\title{
THE GELFAND THEOREM AND ITS CONVERSE IN SASAKIAN GEOMETRY
}

\author{
By O. KowALSKI AND L. VANHECKE
}

\section{Introduction.}

The $\varphi$-symmetric spaces have been introduced in Sasakian geometry by $T$. Takahashi [10] where they seem to be the analogs of the symmetric spaces.

Symmetric spaces have been studied extensively and they have some remarkable properties. In particular, it is well-known that for a symmetric space $M=G / H$, where $G$ denotes the connected component of the identity of the full isometry group $\mathscr{g}(M)$, the algebra $\mathscr{D}(G / H)$ of invariant differential operators is commutative. This is known as the Gelfand theorem. But the converse theorem is not true in general. There are a lot of non-symmetric homogeneous Riemannian manifolds where this algebra is commutative. However, it is shown in [9] that the Hermitian symmetric spaces can be characterized as the homogeneous Kähler manifolds with commutative algebra $\mathscr{D}(G / H)$, where $G$ denotes the connected component of the identity of the group of holomorphic isometries.

The main purpose of this paper is to derive a similar result for $\varphi$-symmetric spaces using here the group of automorphisms of the Sasakian structure. However, we do not obtain a complete commutativity but only commutativity modulo the characteristic differential operator determined by the characteristic vector field.

At the same time we derive a second characterization of $\varphi$-symmetric spaces using the property that all geodesics are orbits of one-parameter subgroups of automorphisms.

\section{Preliminaries.}

A $C^{\infty}$ manifold $M^{2 n+1}$ is said to be an almost contact manifold if the structural group of its tangent bundle is reducible to $U(n) \times 1$. As is well-known, such a manifold admits a tensor field $\varphi$ of type $(1,1)$, a vector field $\xi$ and a one-form $\eta$ satisfying

$$
\eta(\xi)=1, \quad \varphi^{2}=-I+\eta \otimes \xi
$$

Received October 14, 1987 
These conditions imply that $\varphi \xi=0$ and $\eta \circ \varphi=0$. Moreover, $M$ admits a Riemannian metric $g$ satisfying

$$
g(\varphi X, \varphi Y)=g(X, Y)-\eta(X) \eta(Y)
$$

for any tangent vector fields $X$ and $Y$. Note that this implies $\eta(X)=g(X, \xi)$. $M$ together with these structure tensors $(g, \varphi, \eta, \xi)$ is said to be an almost contact metric manifold.

If now these structure tensors satisfy

$$
\left(\nabla_{X} \varphi\right) Y=g(X, Y) \xi-\eta(Y) X,
$$

where $\nabla$ denotes the Riemannian connection of $g$, then $M$ is said to be a Sasakian manifold. This condition implies

$$
\nabla_{X} \xi=-\varphi X
$$

from which it follows that $\xi$ is a Killing vector field. Further, the curvature tensor

$$
R_{X Y} Z=\nabla_{[X, Y]} Z-\left[\nabla_{X}, \nabla_{Y}\right] Z
$$

of a Sasakian manifold satisfies

$$
\begin{aligned}
& R_{X \xi} Y=g(X, Y) \xi-\eta(Y) X, \\
& R_{X Y \xi}=\eta(X) Y-\eta(Y) X .
\end{aligned}
$$

A plane section in $T_{m} M^{2 n+1}, m \in M$, is called a $\varphi$-section if it possesses an orthonormal basis of the form $\{X, \varphi X\}$, where $X \in T_{m} M^{2 n+1}$ is a vector orthogonal to $\xi_{m}$. The sectional curvature $K(X, \varphi X)=H(X)=R(X, \varphi X, X, \varphi X)$ is called the associated $\varphi$-sectional curvature. (We refer to [1], [13] for more details and references about Sasakian geometry.)

A geodesic $\gamma$ on a Sasakian manifold is said to be a $\varphi$-geodesic if $\eta\left(\gamma^{\prime}\right)=0$. It is easy to see that a geodesic which is orthogonal to $\xi$ at one point remains orthogonal to $\xi$.

A local diffeomorphism $s_{m}$ of $M$ defined in a neighborhood $U \subset M$ of the point $m \in M$ is said to be a $\varphi$-geodesic symmetry if for each point $p$ from $U$ which lies on the integral curve of $\xi$ through $m$, and for each $\varphi$-geodesic $\gamma(s)$ such that $\gamma(0)=p$ we have

$$
\left(s_{m} \circ \gamma\right)(s)=\gamma(-s)
$$

for all $s$ with $\gamma( \pm s) \in U, s$ being the arc length. Note that

$$
s_{m *}(m)=-I+2 \eta \otimes \xi \text {. }
$$

T. Takahashi introduced [10] the notion of a locally $\varphi$-symmetric space by requiring that 


$$
\varphi^{2}\left(\nabla_{V} R\right)_{X Y} Z=0
$$

for all vector fields $V, X, Y, Z$ orthogonal to $\xi$. This is equivalent to

$$
\nabla_{V} R_{X Y Z W}=0
$$

for all vector fields $V, X, Y, Z, W$ orthogonal to $\xi$.

Now we state several equivalent properties of (1). First, let $\tilde{U}$ be a neighborhood on $M$ on which $\xi$ is regular. Then, as is well-known, the fibration $\pi: \tilde{U} \rightarrow U=\tilde{U} / \xi$ gives a Kähler structure $(J, G)$ on the base manifold $U$. We have

Proposition 1 [10]. A Sasakian manifold is a locally $\varphi$-symmetric space if and only if each Kähler manifold, which is the base space of a local fibering, is a Hermitian locally symmetric space.

Next, let

$$
\phi(X, Y)=d \eta(X, Y)=g(X, \varphi Y)
$$

Then we have

Proposition 2 [2]. Let $M$ be a Sasakıan manifold. Then $M$ is a locally $\varphi$-symmetric space if and only if the local $\varphi$-geodesic symmetries are
i ) $\phi$-preserving, or
ii) $\varphi$-preserving, or
iii) $\eta$-preserving, or
iv) $g$-preserving.

Finally, the following characterization will prove to be useful. only if

Proposition 3 [3]. A Sasakian manifold is locally $\varphi$-symmetric if and

$$
\nabla_{X} R_{X \varphi X X \varphi X}=0
$$

for all vector fields $X$ orthogonal to $\xi$.

M. Okumura determined on a Sasakian manifold $M$ with structure tensors $(\varphi, \eta, \xi, g)$ a linear connection $\bar{\nabla}$, which is very useful for the study of Sasakian geometry, by

where

$$
\bar{\nabla}_{X} Y=\nabla_{X} Y-T_{X} Y
$$

$$
T_{X} Y=-d \eta(X, Y) \xi+\eta(X) \varphi Y-\eta(Y) \varphi X
$$

The torsion of $\bar{\nabla}$ is $-2 T$ and by direct computation we find easily

$$
\bar{\nabla} g=\bar{\nabla} \phi=\bar{\nabla} \eta=\bar{\nabla} \xi=\bar{\nabla} T=0 \text {. }
$$


Moreover, if $\bar{R}$ denotes the curvature tensor of $\bar{\nabla}$ we have

Proposition 4 [10]. A necessary and sufficient condition for a Sasakian manifold to be locally $\varphi$-symmetric is that $\bar{\nabla} \bar{R}=0$.

In particular, this implies that a locally $\varphi$-symmetric space is locally homogeneous and since (2) implies

$$
g\left(T_{X} Y_{1} X\right)=0,
$$

it follows (see [11]) that $T$ is a naturally reductive structure on $M$. This implies that a complete simply connected locally $\varphi$-symmetric space is a naturally reductive homogeneous space. $\bar{\nabla}$ is the canonical connection with respect to the natural reductive decomposition. As is shown in [3], this leads to

Proposition 5. Let $M$ be a complete, connected, simply connected Sasakian manifold. Then $M$ is a globally $\varphi$-symmetric space if and only if $M$ is a naturally reductive homogeneous space with invariant Sasakian structure.

Note that in [10] a globally $\varphi$-symmetric space $M$ is defined as a Sasakian manifold such that any $\varphi$-geodesic symmetry of $M$ is extendable as a global automorphism of $M$ and such that the Killing vector field $\xi$ generates the oneparameter group $G^{1}$ of global transformations which are automatically automorphisms of $M$. In this case the group $A(M)$ of all automorphisms of $M$ is a transitive Lie transformation group of $M$ and since a homogeneous Sasakian manifold is regular, $M$ is a principal $G^{1}$-bundle over a Kähler manifold $B$, which is a Hermitian symmetric space.

Note also that a complete simply connected Sasakian locally $\varphi$-symmetric space is globally $\varphi$-symmetric [10].

\section{3. $\varphi$-Geodesics and one-orbits of automorphisms.}

In this section we give a new characterization of $\varphi$-symmetric spaces using $\varphi$-geodesics. We start with

THEOREM 6. A Sasakian manifold $M$ is a locally $\varphi$-symmetric space if and only if the $\varphi$-sectional curvature $H\left(\gamma^{\prime}\right)$ is constant along the $\varphi$-geodesic $\gamma$ for all $\gamma$.

Proof. Let $\gamma$ be a $\varphi$-geodesic. Then $H\left(\gamma^{\prime}\right)$ is constant along $\gamma$ if and only if

$$
\gamma^{\prime}\left(R_{\gamma^{\prime} \varphi \gamma^{\prime} \gamma^{\prime} \varphi \gamma^{\prime}}\right)=0 \text {. }
$$

This is equivalent to

$$
\nabla_{\gamma^{\prime}} R_{\gamma^{\prime} \varphi \gamma^{\prime} \gamma^{\prime} \varphi \gamma^{\prime}}+2 R_{\gamma^{\prime}\left(\nabla_{\gamma^{\prime}} \varphi\right) \gamma^{\prime} \gamma^{\prime} \varphi \gamma^{\prime}}=0
$$

But, using the formulas of section 2 , we have 
and

$$
\left(\nabla_{\gamma^{\prime}} \varphi\right) \gamma^{\prime}=\xi
$$

So (3) is equivalent to

$$
R_{\gamma^{\prime} \xi} \gamma^{\prime}=\xi
$$

$$
\nabla_{\gamma^{\prime}} R_{\gamma^{\prime} \varphi r^{\prime} \gamma^{\prime} \varphi \gamma^{\prime}}=0
$$

and hence $\nabla_{X} R_{X \varphi X X \varphi X}=0$ for any $X$ orthogonal to $\xi$. Now the result follows from Proposition 3.

From this we obtain

COROLLARY 7. Let $M$ be a Sasakian manifold and suppose that each $\varphi$-geodesic is an orbit of a one-parameter group of automorphisms of the Sasakian structure. Then $M$ is a locally $\varphi$-symmetric space.

Proof. We just have to note that $H\left(\gamma^{\prime}\right)$ is constant along any $\varphi$-geodesic $\gamma$.

Note that the converse follows from the properties given in section 2: On each complete simply connected locally $\varphi$-symmetric space, each $\varphi$-geodesic is an orbit of a one-parameter subgroup of automorphisms of the Sasakian structure. In fact, each geodesic is such an orbit.

Corollary 7 provides a new proof for a part of Proposition 5.

\section{Invariant differential operators and the Gelfand theorem.}

Let $M=G / H$ be a homogeneous space where $G$ is a connected Lie group and $H$ a closed subgroup. A differential operator $D$ on $M$ is said to be invariant with respect to $G$ if

$$
(D f) \circ g=D(f \circ g)
$$

for all $g \in G$ and all smooth functions $f$ with compact support.

A well-known result by Gelfand states that each Riemannian globally symmetric space $(M, g)$, for which $I_{0}(M)$ is the identity component of the full group of isometries, has the property that the algebra of all $I_{0}(M)$-invariant differential operators on $M$ is commutative. But there are also a lot of nonsymmetric Riemannian homogeneous spaces with the same property. In particular, all naturally reductive spaces of dimension $\leqq 5$ have this property. (This is no longer true for higher dimensions, as has been shown in [6].)

Further, we proved in [9] a converse for the Gelfand theorem in the framework of Kähler geometry (see also [12]):

Proposition 8 [9]. Let $(M, g, J)$ be a simply connected homogeneous Kähler manifold. Then $(M, g, J)$ is Hermitian symmetric if and only if the algebra of invariant differential operators, with respect to the identity component of the group of holomorphic isometries, is commutative. 
Remark. It has been shown in [7], [8] that naturally reductive spaces $M$ in dimension 3 and 5 are always "commutative" with respect to $I_{0}(M)$. Hence the globally $\varphi$-symmetric spaces of dimension 3 and 5 are commutative with respect to $I_{0}(M)$. We do not know if this is true for arbitrary dimension $2 n+1 \geqq 7$.

In what follows let $M$ be a homogeneous Sasakian manifold with structure tensors $(g, \varphi, \eta, \xi)$. Then $M$ is a reductive homogeneous space, $M=G / H$, where $G=B_{0}(M)$, the identity component of the full automorphism group $B(M)$ of the Sasakian structure and $H$ is the isotropy subgroup at the origin $p \in M$. Consider the algebra $\mathscr{D}(G / H)$ of all $G$-invariant differential operators on $M=G / H$. Each transformation $\alpha \in G$ preserves the canonical vector field $\xi$, and hence $\xi$ can be interpreted as an element of $\mathscr{D}(G / H)$.

We are going to prove the following Sasakian version of the Gelfand theorem and its converse:

THEOREM 9. Let $M$ be a simply connected homogeneous Sasakian manifold. Then $M$ is a globally $\varphi$-symmetric space if and only if the algebra $\mathscr{D}(G / H)$ is commutative modulo $\xi$.

Proof. First, we know that the homogeneous Sasakian manifold is a principal $G^{1}$-bundle over a Kähler manifold $\tilde{M}$, where $G^{1}$ is a one-dimensional Lie group isomorphic to the one-parameter group of global transformations generated by $\xi$. Let $A(\tilde{M})$ be the group of all automorphisms of the Kähler manifold $\tilde{M}$. Obviously, $G^{1}$ is a Lie subgroup of $G$ and it belongs to the center of $B(M)$. Hence $\tilde{G}=G / G^{1}$ is a Lie group isomorphic to a subgroup of $A(\tilde{M})$. If we identify $\tilde{G}$ with this subgroup (which acts transitively on $\tilde{M}$ ), $\tilde{M}$ can be written in the form $\tilde{M}=\tilde{G} / \tilde{H}$, where $\tilde{H}$ is the isotropy subgroup at $x=\pi(p) \in \tilde{M}$. The projection $G \rightarrow \tilde{G}$ induces an isomorphism of $H$ onto $\widetilde{H}$ because $H \cap G^{1}=\{e\}$ in $G$.

Consider now the algebra $\mathscr{D}(\tilde{G} / \tilde{H})$ of $\tilde{G}$-invariant differential operators on $\tilde{M}$. We prove

Proposition 10. There is a canonical projection (an algebra homomorphism onto) $\rho: \mathscr{D}(G / H) \rightarrow \mathscr{D}(\tilde{G} / \widetilde{H})$ with kernel $\mathscr{D}(G / H) \xi$.

Proof. Consider an $\operatorname{ad}(H)$-invariant decomposition $g=m+h$ and an $\operatorname{ad}(\widetilde{H})$ invariant decomposition $\tilde{g}=\tilde{m}+\tilde{h}$ (such decompositions always exist). According to [4], we can obtain the operator algebra $\mathscr{D}(G / H)$ in a purely algebraic way, as follows: Let $S(m)$ be the algebra of all polynomial functions on the dual space $m^{*}$ of $m$. (For any basis $\left\{X_{1}, \cdots, X_{n}\right\}$ of $m, S(m)$ can be identified with the polynomial ring $R\left[X_{1}, \cdots, X_{n}\right]$.) Denote by $\mathscr{D}(G)$ the algebra of all leftinvariant differential operators on $G$ (this can be identified with the universal enveloping algebra $U(g)$ ). We can introduce the operation $\lambda: S(m) \rightarrow \mathscr{D}(G)$, called "symmetrization", as follows: for any vectors $Y_{1}, \cdots, Y_{p}$ selected from the basis $\left\{X_{1}, \cdots, X_{n}\right\}$ of $m$ put 


$$
\lambda\left(Y_{1} Y_{2} \cdots Y_{p}\right)=\frac{1}{p !} \sum_{\sigma} Y_{\sigma(1)} \cdot Y_{\sigma(2)} \cdots Y_{\sigma(p)} \in \mathscr{D}(G)
$$

(where $\sigma$ is running over all permutations of $\{1, \cdots, p\}$ ) and extend this map to the whole of $S(m)$ by the linearity. It is known that $\lambda$ is an injective linear map. Now, all elements of the algebra $\mathscr{D}(G)$ determine some differential operators on $G / H$. If we denote by $I(m) \subset S(m)$ the subring of all $\operatorname{ad}(H)$-invariant polynomial functions, then $\lambda(I(m))$ can be identified with $\mathscr{D}(G / H)$ via a bijection.

On the other hand, a generator $Z$ of the one-dimensional central subalgebra $g^{1} \subset g$ determines the vector field $\xi$ as an element of $\mathscr{D}(G / H)$. Hence the decomposition $g=m+h$ can always be chosen such that $g^{1} \subset m$, and we can write $m=m^{\prime}+g^{1}$ (an orthogonal decomposition with respect to the scalar product $\langle$,$\left.\rangle on m \cong T_{p} M\right)$. In particular we get $\operatorname{ad}(H) m^{\prime} \subset m^{\prime}$ because $\operatorname{ad}(H)$ preserves the scalar product.

We can consider an analogous construction for the algebra $\mathscr{D}(\tilde{G} / \tilde{H})$ using the $\operatorname{ad}(\tilde{H})$-invariant decomposition $\tilde{g}=\tilde{m}+\tilde{h}$. Now, consider the projection $\alpha: g \rightarrow \tilde{g}$ with the kernel $g^{1}$. Then $\alpha$ maps isomorphically $m^{\prime}$ onto $\tilde{m}$ and $h$ onto $\tilde{h}$. We obtain a natural isomorphism between $S\left(m^{\prime}\right)$ and $S(\tilde{m})$ and also between $I\left(m^{\prime}\right)$ and $I(\tilde{m})$ (we note that ad $(H)$ acts on $m^{\prime}$ exactly in the same way as $\operatorname{ad}(\tilde{H})$ acts on $\tilde{m})$. Further, $I(m)=I\left(m^{\prime}\right) \oplus I(m) g^{1}$. Finally, the projection $\alpha: g \rightarrow \tilde{g}$ induces a projection $U(\alpha): U(g) \rightarrow U(\tilde{g})$ with the kernel $U(g) g^{1}$ ( $g^{1}$ belongs to the center of $U(g)$ ), i. e., a projection $\mathscr{D}(G) \rightarrow \mathscr{D}(\tilde{G})$ with the kernel $\mathscr{D}(G) \xi$. Now, $\lambda(I(m)) \subset U(g)$ is mapped on the whole of $\lambda(I(\tilde{m})) \subset U(\tilde{g})$ because so is already $\lambda\left(I\left(m^{\prime}\right)\right) \subset \lambda(I(m))$. The fact that the kernel must be $\lambda(I(m)) g^{1}$ is now obvious. Hence our Proposition follows.

Hence we obtain

Corollary 11. The algebra $\mathscr{D}(\tilde{G} / \tilde{H})$ is commutative if and only if $\mathscr{D}(G / H)$ is commutative modulo $\xi$.

Let now $M$ be globally $\varphi$-symmetric and let $s: M \rightarrow M$ be the $\varphi$-symmetry with the origin $p \in M$ as center. Then one can see easily that $\left(G, H \times G^{1}\right)$ forms a symmetric pair with respect to the involution $\sigma: G \rightarrow G$ given by $g \mapsto s \circ g \circ s$. Then $(\tilde{G}, \tilde{H})$ forms a symmetric pair with respect to the involution $\tilde{\sigma}: \widetilde{G} \rightarrow \widetilde{G}$ induced by $\sigma$ via the projection. According to [5, Corollary 5.4, p. 293], the algebra $\mathscr{D}(\tilde{G} / \widetilde{H})$ is commutative and hence $\mathscr{D}(G / H)$ is commutative modulo $\xi$.

Conversely, let $\mathscr{D}(G / H)$ be commutative modulo $\xi$. Then $\mathscr{D}(\tilde{G} / \widetilde{H})$ is commutative and according to [9], the space $\tilde{M}$ is locally Hermitian symmetric. So, Proposition 1 implies that the Sasakian manifold $M$ is locally $\varphi$-symmetric. Because $M$ is also complete and simply connected, it is globally $\varphi$-symmetric. This completes the proof. 


\section{REFERENCES}

[1] D.E. Blair, Contact manifolds in Riemannian geometry, Lecture Notes in Mathematics, 509, Springer-Verlag, Berlin-Heidelberg-New York, 1976.

[2] D.E. Blair and L. Vanhecke, Symmetries and $\varphi$-symmetric spaces, Tôhoku Math. J. 39 (1987), 373-383.

[3] D.E. Blair and L. Vanhecke, New characterizations of $\varphi$-symmetric spaces, Kodai Math. J. 10 (1987), 102-107.

[4] S. Helgason, Differential geometry and symmetric spaces, Academic Press, New York, 1962.

[5] S. Helgason, Groups and geometric analysis, Academic Press, New York, 1984.

[6] J. A. JIMENEZ, Existence of Hermitian $n$-symmetric spaces and of non-commutative naturally reductive spaces, to appear in Math. $Z$..

[7] O. KowALsKI, Spaces with volume-preserving geodesic symmetries and related classes of Riemannian manifolds, Rend. Sem. Math. Univ. e Politec. Torino, Fascicolo Speciale, Settembre 1983, 131-158.

[8] O. KowAlski AND L. VANHECKE, Classification of five-dimensional naturally reductive spaces, Math. Proc. Cambridge Philos. Soc. 97 (1985), 445-463.

[9] O. KowALSKI AND L. VANHECKE, The Gelfand theorem and its converse for Kähler manifolds, Proc. Amer. Math. Soc., to appear.

[10] T. Takahashi, Sasakian $\varphi$-symmetric spaces, Tôhoku Math. J. 29 (1977), 91-113.

[11] F. TRICERRI AND L. VANHECKE, Homogeneous structures on Riemannian manifolds, London Math. Soc. Lecture Note Series, 83, Cambridge Univ. Press, London, 1983.

[12] L. VANHECKE, Symmetries on homogeneous Kähler manifolds, Differential Geometry and its Applications (Eds. D. Krupka and A. Švec), Reidel Publ. Co., 1987, 339-357.

[13] K. Yano and M. Kon, Structures on manifolds, Series in Pure Mathematics, 3, World Scientific Publ. Co., Singapore, 1984.

Faculty of Mathematics and Physics

Charles University

SOKOLOVSKÁ 83

18600 Praha, Czechoslovakia

Department of Mathematics

Katholieke Universiteit LEUVEN

Celestijnenlaan 200B

B-3030 Leuven, Belgium 